\title{
Riscos em Iniciativas de Melhoria de Processos de Software Aderentes ao MR-MPS-SW e ao CMMI-DEV: Uma Investigação no Contexto Brasileiro
}

\author{
Eliezer Dutra $^{1,2}$, Gleison Santos ${ }^{1}$ \\ ${ }^{1}$ Programa de Pós-Graduação em Informática - Universidade Federal do Estado do Rio \\ de Janeiro (UNIRIO) - Av. Pasteur 458, Urca, CEP 22290-240 - Rio de Janeiro, RJ \\ ${ }^{2}$ Centro Federal de Educação Tecnológica Celso Suckow da Fonseca (CEFET/RJ) \\ Estrada de Adrianópolis, 1317 - Santa Rita, CEP: 25265-000 - Nova Iguaçu, RJ \\ \{eliezer.goncalves, gleison.santos\}@uniriotec.br
}

Resumo. Iniciativas de melhoria de processos de software são afetadas por diversos problemas que prejudicam a institucionalização dos processos. Nem sempre as organizações estão cientes dos possíveis problemas e, assim, não se precaveem para evitá-los. Este artigo apresenta um estudo qualitativo cujo objetivo é identificar riscos que comumente afetam iniciativas de melhoria de processos de software aderentes ao MR-MPS-SW e ao CMMI-DEV. Um mapeamento sistemático foi realizado nos principais eventos científicos que tratam especificamente qualidade de software no Brasil (SBQS e WAMPS). Procedimentos de codificação foram utilizados para identificar e categorizar os problemas relatados. Foram analisados 497 artigos e identificados 1152 códigos. Os códigos foram generalizados e organizados em 134 riscos associados a 34 categorias.

\begin{abstract}
Several problems affect software process improvement initiatives and may hinder software processes institutionalization. Organizations are not always aware of such problems and therefore might be unable to avoid them. We present a qualitative study aiming to identify risks that might affect software process improvement initiatives based on MR-MPS-SW or CMMIDev. We have executed a systematic mapping study on SBQS and WAMPS, which are the main software quality venues in Brazil. We used codification procedures to identify and categorize mentioned problems. We analyzed 497 articles and identified 1152 codes. These codes were later generalized and organized in 134 risks associated to 34 risks categories.
\end{abstract}

\section{Introdução}

A competitividade atual exige das organizações desenvolvedoras de software a implantação de melhorias em seus processos com o objetivo de aumentar a qualidade do produto e diminuir o custo, possibilitando, assim, aumento de rentabilidade. Modelos de maturidade como MR-MPS-SW (SOFTEX, 2012) e CMMI-DEV (CMMI Product Team, 2010) auxiliam na definição e na implementação de iniciativas de melhoria de processos de software (MPS).

Vários relatos sobre iniciativas de MPS alertam para baixos níveis de aprovação ou sucesso limitado (Niazi et al., 2006; Paulk et al., 2000; Rocha et al., 2005; O'Connor e Coleman, 2007 e Rainer e Hall, 2003). Birk e Pfahl (2002) relatam que muitas vezes 
os programas de melhoria não são tratados como projetos reais, o que dificulta a aplicação de métodos e técnicas comuns à gerência de projetos. No entanto, nem sempre as organizações estão cientes dos possíveis problemas que podem ocorrer em iniciativas de MPS e, muitas vezes, não têm como evitá-los. Iniciativas de MPS devem ser conduzidas como projetos reais, utilizando as boas práticas de gestão de projetos, incluindo tratamento de riscos. A não identificação dos riscos que podem afetar uma iniciativa de MPS pode prejudicar a institucionalização dos processos ou até levar ao seu cancelamento.

Desta forma, este artigo apresenta os resultados parciais da execução de um estudo qualitativo, baseado em um mapeamento sistemático (Kitchenham e Charters, 2007), cujo objetivo é apresentar, através de procedimentos de codificação (Corbin e Strauss, 2008), um conjunto de riscos que afetam iniciativas brasileiras de MPS baseadas nos modelos de maturidade MR-MPS-SW e CMMI-DEV. Os problemas relacionados à implantação de MPS relatados nos anais do Simpósio Brasileiro de Qualidade de Software (SBQS) e do Workshop Anual do MPS.BR (WAMPS) desde 2004 e 2005, respectivamente, foram identificados e categorizados gerando 1152 códigos diferentes. Estes fóruns foram escolhidos devido ao fato de serem os principais eventos científicos que tratam especificamente qualidade de software no Brasil. Além disso, possuem grande número de publicações contendo relatos de experiência relacionados à implantação de MPS em organizações de software. Isso torna a análise dessas fontes relevante, pois esses artigos foram escritos por consultores, membros da indústria e pesquisadores, e permitem, assim, a reconstrução das experiências destas iniciativas de MPS.

Além desta introdução, este trabalho apresenta mais 5 seções: a seção 2 apresenta a fundamentação teórica, a seção 3 expõe a metodologia de pesquisa e a seção 4 apresenta resultados obtidos, enquanto a seção 5 apresenta trabalhos relacionados e, por fim, na seção 6 são discutidos as considerações finais e trabalhos futuros.

\section{Fundamentação Teórica}

\subsection{Riscos em Iniciativas de MPS}

Risco é um evento ou condição incerta que, se ocorrer, provocará um efeito positivo ou negativo em um ou mais objetivos do projeto (PMI, 2013). Iniciativas de melhoria de processos de software (MPS) são confrontadas com uma série de riscos que dificultam o desenvolvimento e implantação do projeto, que em alguns casos, proporciona um pequeno ou nenhum aumento da capacidade de desenvolver softwares com qualidade. Niazi (2012) acredita que um bom entendimento dos riscos é vital para o sucesso das iniciativas de MPS.

Embora os efeitos de uma gestão de riscos ineficaz ou ausente sejam conhecidos (Iversen et al., 2004), existem poucas pesquisas que identificaram riscos propondo ações de tratamento em iniciativas de MPS, por exemplo, (Mendes et al., 2007). São mais comuns trabalhos que apresentam fatores críticos de sucesso ou insucesso, dificuldades, barreiras ou lições aprendidas, por exemplo, (Rodrigues e Kirner, 2010) e (Niazi et al., 2006).

Niazi (2012) executou um estudo exploratório com profissionais da Austrália para identificação de riscos em MPS. O objetivo do estudo foi obter uma compreensão dos riscos que podem comprometer a implantação de MPS a partir da perspectiva dos 
profissionais envolvidos. Os riscos mais críticos encontrados em Niazi (2012) foram os de políticas organizacionais, falta de apoio, falta de definição de uma metodologia para implantação de MPS, falta de consciência e falta de recursos.

Mendes et al. (2007) identificaram na literatura os riscos mais citados com o intuito de apresentar os dez mais recorrentes em iniciativas de MPS. Além disso, foram propostas ações de controle com o objetivo de mitigar ou contingenciar os riscos. Os dez riscos mais citados foram os de falta de compromisso da equipe (gerência sênior, gerências médias e equipe), conhecimento inadequado dos colaboradores de princípios gerais de MPS ou do modelo de maturidade adotado, não alinhamento do programa de MPS com os objetivos organizacionais, recursos humanos qualificados insuficientes, resistência cultural da organização na implantação de MPS, falta de envolvimento da equipe da empresa, indisponibilidade de tecnologia de apoio às atividades de gerência e desenvolvimento, expectativas não realistas da gerência, perda de apoio financeiro ao programa MPS e tecnologia a ser usada para guiar o processo de MPS.

\subsection{Conceitos Relacionados à Identificação de Riscos}

A norma internacional de Gestão de Riscos ISO 31000 (ISO, 2009) propõe princípios e diretrizes genéricas para o gerenciamento de riscos, definindo, ainda, um processo de identificação de riscos como uma metodologia de busca, reconhecimento e descrição dos riscos. Contudo, Falbo (2010) afirma que não há um consenso na literatura a respeito da estrutura de um plano de riscos ou de como um risco deve ser detalhado. Para auxiliar o reconhecimento dos conceitos necessários para um processo de identificação de riscos e de ações de tratamento, procurou-se olhar a literatura técnica a respeito para criação de um modelo conceitual. Foram consultados a norma internacional sobre gestão de riscos ISO 31000 (ISO, 2009), o CMMI-DEV (CMMI Product Team, 2010), o MR-MPS-SW (SOFTEX, 2012), o PMBOK (PMI, 2013), e uma ontologia de gestão de riscos (Falbo, 2010).

O PMBOK (PMI, 2013) propõe a descrição de riscos através de categorias, fontes, causas e impactos. O resultado do processo de identificação é uma lista de riscos descritos com o maior detalhamento possível. Para isso, o PMBOK propõe o registro dos riscos por meio da seguinte estrutura para descrição dos riscos: "o evento pode ocorrer, causando o impacto, ou, se uma causa existe, o evento pode ocorrer, levando ao efeito". Para o CMMI-DEV (CMMI Product Team, 2010) e o MR-MPS-SW (SOFTEX, 2012) a descrição do risco deve envolver contexto, condições, consequência, fontes e categorias. Falbo (2010) apresenta os principais conceitos relacionados à gestão de riscos, incluindo risco, causa, consequência, categoria e fontes, entre outros, organizados em uma ontologia, cujo objetivo é propor uma referência de um vocabulário comum para o domínio de riscos. A ISO 31000 (ISO, 2009) apresenta o processo de identificação de riscos através do estabelecimento das fontes de riscos, eventos e suas causas e consequências potenciais, tendo por finalidade gerar uma lista abrangente de riscos baseada nos eventos. A ISO 31000 também relata que um risco é muitas vezes caraterizado pela referência aos eventos. Tanto o PMBOK quanto a ISO 31000 apresentam a descrição de um risco baseada em um evento e, de acordo com a ISO 31000, um risco pode ter um ou mais eventos associados.

Dessa forma, os elementos essenciais para identificação de riscos e ações de controle apresentados no CMMI-DEV, MR-MPS-SW, PMBOK, ISO 31000 e na ontologia proposta por Falbo (2010) podem ser sumarizados no modelo conceitual 
parcial apresentado na Figura 1. Este modelo mostra que:

- Um EVENTO tem CAUSA e CONSEQUÊNCIA associadas, é tratado por meio de uma AÇÃO que resulta em um EFEITO.

- Um EVENTO está associado a um RISCO que, por sua vez, também tem CAUSA, CONSEQUÊNCIA e AÇÃO associados.

- Um RISCO está associado a uma CATEGORIA de risco.

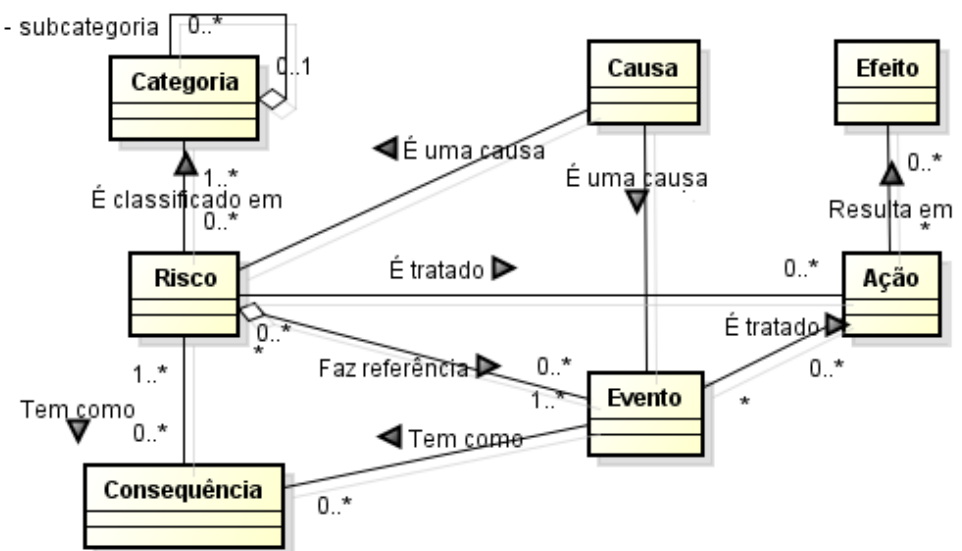

Figura 1. Modelo conceitual para identificação de riscos e ações de controle

Destaca-se que a estrutura proposta na Figura 1 tem o objetivo de nortear a identificação dos riscos neste estudo qualitativo e não representa todos os elementos possíveis de serem utilizados para identificação ou gestão de riscos.

\section{Metodologia da Pesquisa}

\subsection{Metodologia}

O estudo qualitativo descrito neste artigo tem por objetivo identificar riscos, causas, consequências, ações de controle e os efeitos destas ações em iniciativas brasileiras de melhoria de processos de software (MPS) baseadas nos modelos de maturidade MRMPS-SW e CMMI-DEV. Dessa forma, uma questão de pesquisa foi definida para alcançar o objetivo descrito:

- Que riscos comumente afetam iniciativas de melhoria de processos de software baseadas nos modelos de maturidade CMMI-DEV e MR-MPS-SW?

Para responder a essa pergunta e identificar os riscos da maneira mais completa possível, o processo de identificação de risco adotado pelos pesquisadores também procurou identificar: "O que desencadeou o risco?"; "Que ações foram tomadas para tratar os riscos?" e "Qual o efeito das ações identificadas para tratar os riscos?".

Dessa forma, foi definido um protocolo baseado no mapeamento sistemático (Kitchenham e Charters, 2007) com o objetivo de pesquisar, avaliar e interpretar artigos relevantes para a questão de pesquisa. No âmbito deste estudo foi adotado o seguinte escopo: (i) publicações do SBQS (Simpósio Brasileiro de Qualidade de Software) e do WAMPS (Workshop Anual do MPS.BR); (ii) publicadas no período relacionado entre 2004 e 2014 para o SBQS e entre 2005 e 2014 para o WAMPS. A escolha desses períodos são justificados pelo fato de o modelo de negócio baseado no uso do MRMPS-SW ter sido criado em 2004 e o I Encontro de Implementadores, evento que 
originou o WAMPS, ter sido realizado em 2005 (mesmo ano da publicação da primeira versão do Guia Geral do MR-MPS-SW). A escolha desses eventos como fonte de dados se torna relevante, pois eles representam os principais fóruns no Brasil que unem a indústria e a academia para discutir problemas e soluções acerca de Qualidade de Software. Além disso, são comuns nestes dois eventos publicações relacionadas a relatos de experiências de implementação de melhoria de processos de software.

Também foram definidos os seguintes critérios de inclusão no escopo do mapeamento sistemático: (i) só serão considerados relatos de experiências e artigos técnicos relacionados a uma iniciativa de MPS baseada nos modelos de maturidade MRMPS-SW ou CMMI-DEV e (ii) que descrevam riscos, dificuldades, problemas, barreiras, lições aprendidas ou ações negativas que influenciaram o projeto e/ou a implantação dos processos. O critério de exclusão definido foi: não serão consideradas as publicações em que todas as proposições apresentem dificuldades, problemas, barreiras ou ações negativas escritas com a linguagem subjetiva e de forma implícita.

A inclusão dos trabalhos no escopo de pesquisa deu-se após a leitura e interpretação de artigos em que foram relatadas circunstâncias que influenciaram negativamente uma iniciativa de MPS. É importante destacar que todos os artigos do período considerado foram lidos e avaliados, por isso não foi necessário definir uma string de busca fixa. Apesar de não usual, o não uso de uma string de busca (ver, por exemplo, (Santos, 2011)) procura evitar uma possível ameaça à validade relacionada ao fato de não se considerar artigos relevantes que não mencionasse palavras-chave relacionadas ao objetivo da pesquisa (como, por exemplo, riscos, melhoria de processos de software, barreiras etc.) no título ou resumo/abstract. O uso de uma string fixa deixaria de identificar muitos problemas, principalmente nos relatos de experiência.

\subsection{Coleta de dados}

Durante a fase de seleção dos trabalhos para o escopo do mapeamento sistemático, o título e o resumo foram lidos para avaliar se o artigo tratava de relatos ou trabalhos técnicos envolvendo iniciativas de MPS baseado nos modelos CMMI-DEV ou MRMPS-SW (critério de inclusão 1). Caso positivo, os artigos eram lidos por completo à procura de proposições que representassem um possível risco, dificuldade, problema ou barreira (critério de inclusão 2). O critério de exclusão não foi aplicado a nenhum artigo.

Após isso, a rede de argumentação utilizada no artigo era analisada para estabelecer um evento que representasse o risco, dificuldade, problema ou barreira principal da proposição. Destaca-se que alguns artigos descreveram problemas como oportunidades e alguns textos utilizaram eufemismo. Assim, para evitar um possível viés, as expressões que destacavam oportunidades ou lições aprendidas sem um significado negativo não foram codificadas.

Em seguida, uma possível relação entre causa e consequência era estabelecida, caso o autor indicasse a origem ou a consequência. Neste momento, códigos foram gerados para cada proposição relevante identificada. Dessa forma, os elementos essenciais identificados no modelo proposto na Figura 1 (evento, causa, consequência, ação e efeito) foram utilizado para apoiar a codificação. Cada código foi associado a um dos elementos do modelo conceitual.

Assim, através do procedimento de codificação (Corbin e Strauss, 2008), os eventos, as causas, as consequências, as ações de tratamento e os efeitos foram 
identificados e um possível relacionamento entre eles foi estabelecido. A transcrição proposta na Figura 2 foi extraída de um relato de experiência (Rocha et al., 2005). As expressões sublinhadas representam os conceitos que originaram os códigos "[EV085]", "[CA134]" e "[AC187]". Para cada código o pesquisador indicou uma descrição baseado no texto do artigo, conforme a seguir: "[EV085] Baixo conhecimento dos conceitos de Engenharia de Software", "[CA134] Nível baixo de formação em informática da equipe da empresa (Universidades fracas e maioria não graduados)" e "[AC187] Capacitar a equipe nos procedimentos, métodos e técnicas utilizadas para apoiar o processo de desenvolvimento". Durante o processo de codificação, um possível relacionamento entre "[CA134]", o evento "[EV085]" e a ação "[AC187]" foi estabelecido. É importante destacar que essas relações foram inferidas a partir das proposições escritas nos artigos e não deduções do pesquisador. Destaca-se, ainda, que os códigos de primeiro nível, ou seja, aqueles diretamente ligados às marcações, tiveram o objetivo de descrever os códigos de forma contextualizada e não apenas criar categorias genéricas neste momento da codificação.

$$
\begin{aligned}
& \text { Pode ser destacada como a maior deficiência nas competências necessárias a equipe da } \\
& \text { empresa o pouco conhecimento em Engenharia de Software [EV085]. Uma vez } \\
& \text { encontrada esta dificuldade na empresa, a maioria dos procedimentos, métodos e técnicas } \\
& \text { utilizadas como apoio ao processo de desenvolvimento precisavam ser ensinados, por } \\
& \text { exemplo, como elaborar descriçóes de casos de uso, diagramas de classes e } \\
& \text { especificações de requisitos, etc. [AC187] Esta dificuldade está relacionada ao nível } \\
& \text { muito baixo de formação em informática da equipe da empresa (universidades fracas e } \\
& \text { maioria de não graduados) } \\
& \text { [CA134]. }
\end{aligned}
$$

Figura 2. Exemplo de codificação de primeiro nível

O processo de codificação foi realizado por dois pesquisadores e teve duração aproximada de 1 ano. Bandeira-de-Melo e Cunha (2010) relatam que pesquisadores inexperientes devem ser advertidos que o projeto de pesquisa absorve o pesquisador: ele deve imergir no mundo dos pesquisados e, ao mesmo tempo, manter distanciamento necessária para que o conhecimento surja dos dados. Isso, demanda tempo e dedicação. Assim, com intuito de analisar a qualidade do processo de codificação, reuniões periódicas foram realizadas entre os pesquisadores. Além de discussões sobre a interpretação dos textos, grafos mostrando o relacionamento entre os códigos (ver Figura 3) eram analisados para verificar as marcações e as relações entre os códigos. Em caso de divergências de interpretação, o texto do artigo era novamente analisado e notas textuais eram desenvolvidas para justificar o caminho interpretativo escolhido.

Após a leitura e codificação de primeiro nível em todos os artigos, iniciou-se o processo de identificação de categorias. Assim, cada evento era analisado através da sua descrição e uma categoria provisória, isto é, risco, era estabelecida. Esse procedimento é iterativo e foi realizado para todos os conceitos propostos na Figura 1: riscos, eventos, causas, consequências, ações e efeitos. Durante as iterações, novas categorias surgiam e as descrições das categorias eram ajustadas.

A Figura 3 apresenta uma parte do processo de codificação. Analisando o "[EV085]" é possível verificar os números (3-5), o primeiro número faz referência à quantidade de citações que esse código obteve e o segundo à quantidade de relacionamentos com outros códigos. Também é possível observar notas denominadas "Contexto - WAMPS (Rocha et al., 2005)", "Contexto - WAMPS (Amaral, 2008)" e Contexto - WAMPS (Mello e Rocha, 2009)" indicando os artigos que originaram o 
código.

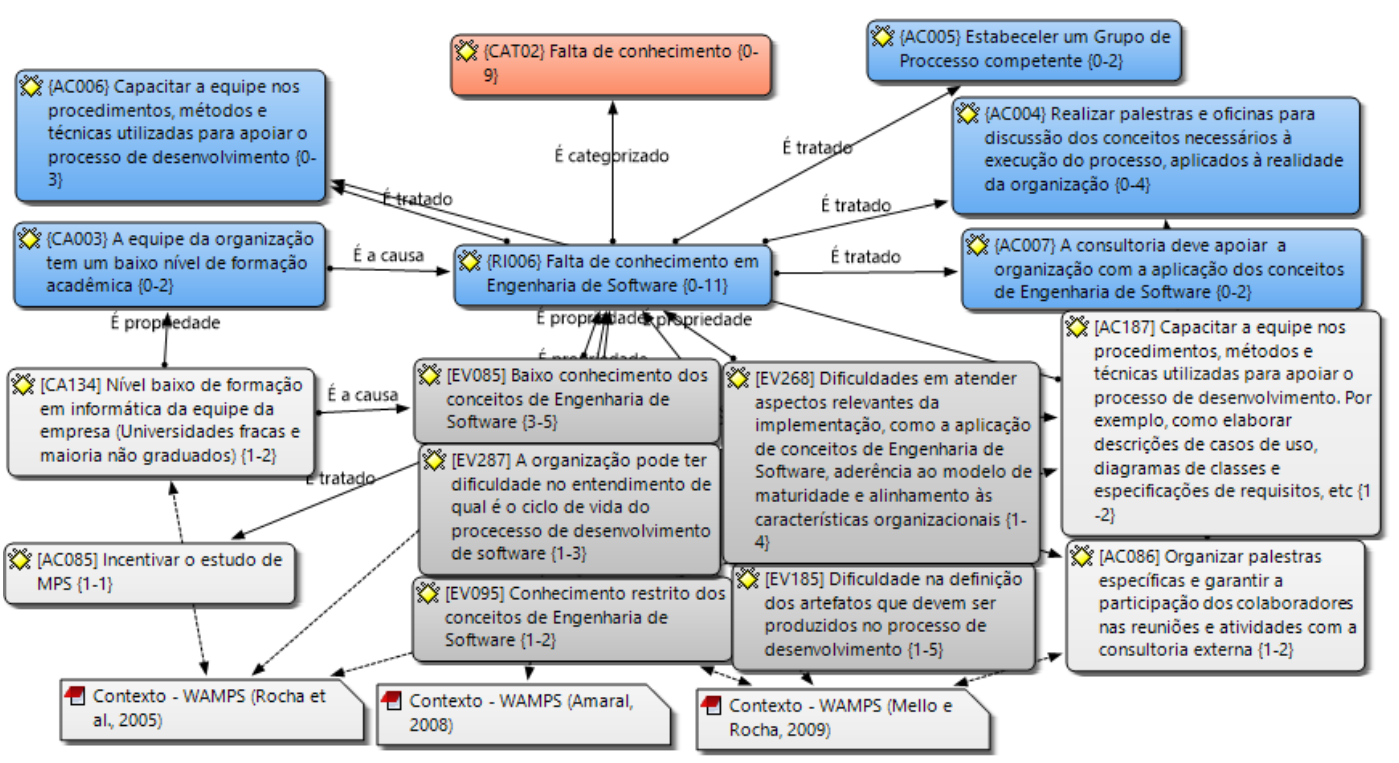

Figura 3. Exemplo de codificação com a identificação de categorias

Dessa forma, os eventos foram categorizados em riscos e os riscos em categorias de riscos. Por exemplo, observe na Figura 3 que os eventos ("[EV085]", "[EV095]", "[EV185]", "[EV268]" e "[EV287]") foram categorizados no risco " $\{$ RI006 $\}$ Falta de conhecimento em Engenharia de Software" que, por sua vez, foi categorizado em " $\{\mathrm{CAT} 02\}$ Falta de conhecimento". Esse processo também foi realizado para as causas, consequências, ações e efeitos. Assim, o risco " $\{$ RI006 $\}$ " foi associado com a causa " $\{\mathrm{CA} 003\} "$ e com as ações " $\{\mathrm{AC} 004\} ", "\{\mathrm{AC} 006\} ", "\{\mathrm{AC} 005\} " \mathrm{e}$ " $\{\mathrm{AC} 007\} "$

Alguns relatos de experiência utilizaram palavras com o sentido figurado. Assim, essas expressões não foram usadas no sentido original, abrindo um caminho para a linguagem subjetiva e, consequentemente, permitindo várias interpretações. Por exemplo, "Durante a execução do projeto houve a saída de pessoas chave, o que resultou no 'esfriamento' do projeto. Assim, faz-se necessário o envolvimento de diversas pessoas nas atividades de definição do processos para que o conhecimento seja disseminado e que a rotatividade não impacte o projeto" (Mendes et al., 2011). Nesta transcrição, a expressão "houve a saída de pessoas chave" foi codificada como um evento, pois apesar da palavra "chave" não ter sido utilizada em seu sentido original, a expressão "pessoas-chave" permite uma interpretação única, cujo o significado é conhecido. Entretanto, a palavra "esfriamento" não foi codificada, pois representa um termo escrito com uma linguagem subjetiva e de forma implícita.

É importante destacar que o artigo entraria no critério de exclusão se todas as proposições (dificuldades, problemas, barreiras ou ações negativas) fossem escritas com linguagem figurada e implícita. Todavia, nenhum artigo foi excluído do mapeamento pela aplicação deste critério de exclusão.

\subsection{Auditoria}

Para avaliar o presente trabalho, foram realizadas duas auditorias. A primeira auditoria teve o objetivo de verificar a codificação de primeiro nível e a segunda o agrupamento dos eventos em riscos e dos riscos em categorias. 
Na primeira auditoria, foi verificado (i) se o código associado (causa, evento, consequência, ação de tratamento ou efeito) estava fundamentado em alguma proposição do artigo, (ii) se existia uma possível relação proposta no artigo para fundamentar a associação entre os códigos (causa, o evento, a consequência e ação de tratamento). Para isso, foi emitido um relatório a partir da ferramenta utilizada (Atlas.ti) para realizar a codificação com todas as marcações (citações originais dos artigos) e os códigos identificados. O segundo relatório apresentava os eventos, suas causas e consequências. $\mathrm{O}$ arquivo físico do Atlas.ti também foi disponibilizado. Esta etapa foi realizada por três pesquisadores que possuem artigos utilizando procedimentos de codificação (Corbin e Strauss, 2008) e também conhecimento dos artigos considerados no escopo da pesquisa.

Ao final, os auditores indicaram algumas discrepâncias entre a marcação original e o código identificado. Algumas dessas discrepâncias foram encontradas pela impossibilidade de o software realizar marcações em diferentes partes do artigo para justificar o entendimento do código sem contabilizar outra ocorrência do código. Outros apontamentos foram sinalizados devido às quebras de páginas dos parágrafos ou das tabelas que continham as marcações, prejudicando assim, a marcação completa da citação. Um auditor questionou a marcação de um mesmo código diversas vezes em um único artigo, essa ocorrência foi esclarecida pelo fato de alguns artigos apresentarem relatos de diferentes iniciativas de MPS em várias organizações.

A segunda auditoria foi realizada por um consultor experiente em iniciativas de MPS. O objetivo desta auditoria foi verificar a coerência dos agrupamentos dos eventos em riscos e os agrupamentos dos riscos em categorias de riscos. Para isso, foi emitido um relatório contendo (i) riscos e eventos associados e (ii) categorias de risco e riscos associados.

Todas as indicações sinalizadas nas duas auditorias foram verificadas e os ajustes necessários foram realizados.

\section{Resultados}

Com a execução do protocolo do mapeamento sistemático, foram analisadas 497 publicações do WAMPS e do SBQS. Desses artigos, 87 foram consideradas parte do escopo após aplicação dos critérios de inclusão e exclusão. Foram identificados 1152 códigos: 398 eventos, 159 causas, 173 consequências, 302 ações de tratamento e 120 efeitos. Os eventos foram agrupados em riscos e os riscos foram agrupados em categorias de risco. Ao final dessa codificação, foram identificados 134 riscos em 34 categorias. A Tabela 1 apresenta todas as categorias de riscos identificadas. Os números apresentados entre parênteses após a descrição, representam, respectivamente, a quantidade total de citações encontradas e o percentual de citações.

A partir dos resultados apresentados na Tabela 1, pode-se perceber que 5 das 34 categorias investigadas compreendem $41,6 \%$ de todas as citações encontrados. Estas foram, portanto, consideradas as categorias de risco que mais influenciaram negativamente a condução de iniciativas de melhoria consideradas. Assim, as 5 categorias mais críticas apontadas neste estudo foram: " $\{$ CAT06 $\}$ Falta de comprometimento ou apoio (10,6\%)", " $\{$ CAT09\} Resistência ao processo ou a iniciativa de MPS (9,8\%)", " $\{$ CAT07 $\}$ Falta de recursos humanos $(7,5 \%)$ ", " $\{$ CAT02 $\}$ Falta de conhecimento (7,3\%)" e " $\{$ CAT01 $\}$ Falta de ferramentas $(6,4 \%)$ ". 
Tabela 1. Categorias de Riscos

\begin{tabular}{|c|c|}
\hline$\{$ CAT01 $\}$ Falta de ferramentas $(33-6,4 \%)$ & $\begin{array}{l}\{\text { CAT18\} Riscos na implantação ou } \\
\text { institucionalização do processo de software }(28 \text { - } \\
5,4 \%)\end{array}$ \\
\hline$\{$ CAT02 $\}$ Falta de conhecimento $(76-7,3 \%)$ & $\{$ CAT19\} Gerência de Projetos (GPR) $(12-2,3 \%)$ \\
\hline$\{$ CAT03 $\}$ Falta de treinamento $(6-1,2 \%)$ & $\{$ CAT20 $\}$ Gerência de Requisitos $(11-2,1 \%)$ \\
\hline$\{$ CAT04\} Falta de comunicação $(6-1,2 \%)$ & $\{$ CAT21\} Gerência de Configuração (GCO) $(2-1,0 \%)$ \\
\hline $\begin{array}{l}\{\text { CAT05\} Falta de recursos financeiros (17 - } \\
3,3 \%)\end{array}$ & $\begin{array}{l}\{\text { CAT } 22\} \text { Gerência de Portfólio de Projetos (GPP) (2 - } \\
0,4 \%)\end{array}$ \\
\hline $\begin{array}{l}\{\text { CAT06 }\} \text { Falta de comprometimento ou apoio } \\
(55-10,6 \%)\end{array}$ & \{CAT23\} Garantia da Qualidade (GQA) (14 - 2,7\%) \\
\hline $\begin{array}{l}\{\text { CAT07 }\} \text { Falta de recursos humanos }(39- \\
7,5 \%)\end{array}$ & $\{$ CAT24\} Medição (MED) $(24$ - 4,6\%) \\
\hline $\begin{array}{l}\{\text { CAT08\} Rotatividade dos colaboradores (14 - } \\
2,7 \%)\end{array}$ & $\begin{array}{l}\{\text { CAT25\} Gerência de Recursos Humanos (GRH) (1 - } \\
0,2 \%)\end{array}$ \\
\hline $\begin{array}{l}\{\text { CAT09\} Resistência ao processo ou a } \\
\text { iniciativa de MPS }(51-9,8 \%)\end{array}$ & \{CAT26\} Gerência de Reutilização (GRU) (15 - 2,9\%) \\
\hline $\begin{array}{l}\{\text { CAT10 }\} \text { Cancelamento da iniciativa (4 - } \\
0,8 \%)\end{array}$ & $\{$ CAT27\} Integração do Produto (ITP) $(1-0,2 \%)$ \\
\hline $\begin{array}{l}\text { \{CAT11\} Riscos oriundos da estrutura } \\
\text { organizacional da instituição }(17-3,3 \%)\end{array}$ & $\{$ CAT28\} Validação (VAL) $(3-0,6 \%)$ \\
\hline $\begin{array}{l}\{\text { CAT12 }\} \text { Riscos no planejamento da iniciativa } \\
\text { de MPS }(23-4,4 \%)\end{array}$ & $\begin{array}{l}\{\text { CAT29\} Projeto e Construção do Produto (PCP) (1 - } \\
0,2 \%)\end{array}$ \\
\hline $\begin{array}{l}\{\text { CAT13\} Riscos da estratégia de implantação } \\
\text { com métodos ágeis }(9-1,7 \%)\end{array}$ & $\{$ CAT30 $\}$ Verificação (VER) $(3-0,6 \%)$ \\
\hline $\begin{array}{l}\{\text { CAT14 }\} \text { Riscos da estratégia de implantação } \\
\text { para grupos cooperados }(11-2,1 \%)\end{array}$ & $\{$ CAT31\} Gerência de Decisões (GDE) $(3-0,6 \%)$ \\
\hline $\begin{array}{l}\{\text { CAT15\} Riscos da definição dos treinamentos } \\
(4-0,8 \%)\end{array}$ & $\{$ CAT32\} Gerência de Riscos (GRI) $(2-0,4 \%)$ \\
\hline $\begin{array}{l}\{\text { CAT16 }\} \text { Planejamento inadequado dos } \\
\text { recursos humanos }(19-3,7 \%)\end{array}$ & $\begin{array}{l}\{\text { CAT33\} Riscos oriundos da preparação para } \\
\text { avaliação }(20-3,9 \%)\end{array}$ \\
\hline $\begin{array}{l}\{\text { CAT17\} Definição inadequada do processo } \\
(20-3,9 \%)\end{array}$ & $\{$ CAT34 $\}$ Riscos oriundos da avaliação $(8-1,5 \%)$ \\
\hline
\end{tabular}

Estas mesmas categorias também foram identificas por Niazi (2012) e Mendes et al. (2007). Rodrigues e Kirner (2010) analisaram essas categorias de risco como dificuldades que influenciam negativamente as iniciativas de MPS. Por isso e, também, devido à restrição de espaço deste artigo, se torna inviável apresentar e analisar todos os códigos identificados referentes aos riscos, eventos, causas, consequências, ações e efeitos. Portanto, serão apresentados e analisados os riscos referentes à definição, implantação e institucionalização dos processos de software "\{CAT17\}" e " $\{$ CAT18\}" e os riscos técnicos relacionados aos processos do níveis G e F do MR-MPS-SW

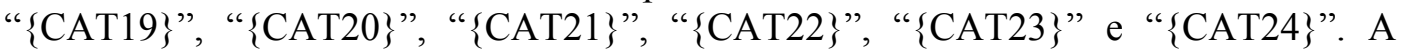
análise dessas categorias é relevante, pois a quantidade de avaliações oficiais nos dois níveis iniciais do MR-MPS-SW perfazem um total de 87\% das avaliações (SOFTEX, 2015).

Nesse contexto, a seção 4.1 analisa os riscos da definição e implantação de processos e a seção 4.2 analisa os riscos associados aos processos do nível $\mathrm{G}$ e $\mathrm{F}$ do MR-MPS-SW. Para cada categoria será identificado o risco com maior número de citações, assim serão indicadas algumas causas, consequência ou ações de tratamento.

\subsection{Riscos da Definição e Implantação de Processos}

A Tabela 2 apresenta riscos que devem ser considerados durante a definição e 
implantação do processo desenvolvimento de software. Os números entre parênteses após a descrição do risco indicam, respectivamente, a quantidade de eventos associados, a quantidade de citações e o percentual de citações. $\mathrm{O}$ número entre parênteses após a descrição da categoria representa o percentual de citações da categoria.

Tabela 2. Riscos de definição e implantação de processos

\begin{tabular}{|c|c|}
\hline $\begin{array}{c}\text { \{CAT17\} Definição inadequada do processo } \\
(3,9 \%)\end{array}$ & $\begin{array}{c}\text { \{CAT18\} Riscos na implantação ou } \\
\text { institucionalização do processo de software }(5,4 \%)\end{array}$ \\
\hline $\begin{array}{l}\{\text { RI048\} O processo pode ser definido sem } \\
\text { ajuda de consultoria especializada }(1 ; 1 ; 0,2 \%)\end{array}$ & $\begin{array}{l}\{\text { RI049\} A organização pode não conseguir realizar a } \\
\text { implantação do processo no projeto piloto }(2 ; 3 ; \\
0,6 \%)\end{array}$ \\
\hline $\begin{array}{l}\text { \{RI053\} O processo pode ser definido sem um } \\
\text { alinhamento com as necessidades da } \\
\text { organização }(7 ; 8 ; 1,5 \%)\end{array}$ & $\begin{array}{l}\{\text { RI052 }\} \text { O processo definido pode mudar } \\
\text { constantemente }(6 ; 10 ; 2,0 \%)\end{array}$ \\
\hline $\begin{array}{l}\{\text { RI054 }\} \text { O processo pode ser definido sem o } \\
\text { estabelecimento ou a avaliação de todas as } \\
\text { atividades necessárias à sua execução }(2 ; 2 ; \\
0,4 \%)\end{array}$ & $\begin{array}{l}\{\text { RI057\} Dificuldades para realização das avaliações } \\
\text { dos processos organizacionais (treinamento, medição } \\
\text { e análise e gestão de processos })(1 ; 2 ; 0,4 \%)\end{array}$ \\
\hline $\begin{array}{l}\{\text { RI059\} Definir e implantar vários processos } \\
\text { simultaneamente }(2 ; 2 ; 0,4 \%)\end{array}$ & $\begin{array}{l}\text { \{RI085\} Alta direção ou a equipe da organização } \\
\text { podem não interagir de forma apropriada com a } \\
\text { consultoria }(4 ; 5 ; 1 \%)\end{array}$ \\
\hline $\begin{array}{l}\{\text { RI076 }\} \text { Indisponibilidade da equipe }(1 ; 1 ; \\
0,2 \%)\end{array}$ & $\begin{array}{l}\{\text { RI086 }\} \text { O processo definido pode reduzir a } \\
\text { produtividade da equipe }(2 ; 3 ; 0,6 \%)\end{array}$ \\
\hline $\begin{array}{l}\{\text { RI078\} Não definir um processo de } \\
\text { desenvolvimento de software para situações } \\
\text { específicas }(2 ; 2 ; 0,4 \%)\end{array}$ & $\begin{array}{l}\{\text { RI090 }\} \text { Experiências anteriores sem êxito em } \\
\text { iniciativas de MPS }(1 ; 2 ; 0,4 \%)\end{array}$ \\
\hline $\begin{array}{l}\{\text { RI080 }\} \text { O processo pode demorar para ser } \\
\text { definido }(1 ; 1 ; 0,2 \%)\end{array}$ & $\begin{array}{l}\text { \{RI121\} A equipe pode encontrar muita dificuldade } \\
\text { em manter sob controle a evolução dos documentos } \\
\text { do projeto }(1 ; 2 ; 0,4 \%)\end{array}$ \\
\hline $\begin{array}{l}\{\text { RI099\} Falta de objetividade na escolha de } \\
\text { diversas soluções possíveis para o processo }(2 ; \\
2 ; 0,4 \%) \\
\{\text { RI111\} Não criar uma visualização gráfica dos } \\
\text { fluxos das atividades e processos }(1 ; 1 ; 0,2 \%)\end{array}$ & (2) \\
\hline
\end{tabular}

$\mathrm{O}$ risco " $\{\mathrm{RI053}\} \mathrm{O}$ processo pode ser definido sem um alinhamento com as necessidades da organização" possui a maior frequência de citações da categoria " $\{\mathrm{CAT} 17\} "$. Algumas ação de tratamento foram identificadas para este risco, por exemplo: "Realizar o diagnóstico tendo como um dos objetivos coletar metas implícitas da organização que guiarão todo o projeto de MPS" e "Desenvolver um checklist de verificação de alinhamento que deve ser preenchido em todo marco do projeto".

$\mathrm{Na}$ categoria " $\{\mathrm{CAT} 18\}$ Riscos na implantação ou institucionalização do processo de software" o risco mais citado com 7 ocorrências foi o " $\{\mathrm{RI052}\}$ O processo definido pode mudar constantemente". Algumas ações de tratamento foram identificadas, por exemplo: "Disseminar conhecimento dos processos e dos conceitos relacionados" e "Utilizar ferramentas adequadas, como a wiki, na comunicação da equipe".

\subsection{Riscos da Definição e Implantação de Processos Específicos do Nível G e F}

A Tabela 3 representa os riscos de implantação de processos específicos do nível $\mathrm{G}$ e $\mathrm{F}$ do MR-MPS-SW. Os números entre parênteses após a descrição do risco indicam, respectivamente, a quantidade de eventos associados, a quantidade de citações e o percentual de citações. O número entre parênteses após a descrição da categoria 
representa o percentual de citações da categoria.

Tabela 3. Riscos de definição e implantação de processos específicos

\begin{tabular}{|c|c|}
\hline$\{$ CAT19\} Gerência de Projetos (GPR) $(2,3 \%)$ & $\begin{array}{l}\text { \{CAT22\} Gerência de Portfólio de Projetos (GPP) } \\
\qquad(0,4 \%)\end{array}$ \\
\hline $\begin{array}{l}\{\text { RI015\} Falta de conhecimento de práticas de } \\
\text { gerência de projetos }(4 ; 5 ; 1,0 \%)\end{array}$ & $\begin{array}{l}\{\mathrm{RI} 125\} \text { Um único colaborador pode ser responsável } \\
\text { pela análise técnica dos projetos }(1 ; 1 ; 0,2 \%)\end{array}$ \\
\hline $\begin{array}{l}\{\text { RI058\} Dificuldades no entendimento dos } \\
\text { conceitos de planejamento e controle da gestão } \\
\text { de documentos }(1 ; 1 ; 0,2 \%)\end{array}$ & $\begin{array}{l}\text { \{RI126\} A organização pode executar múltiplas } \\
\text { alocações entre recursos dos projetos de } \\
\text { desenvolvimento de software de forma manual }(1 ; 1 ; \\
0,2 \% \text { ) }\end{array}$ \\
\hline $\begin{array}{l}\{\text { RI119\} A organização pode não registrar } \\
\text { problemas ou ações para corrigir os desvios dos } \\
\text { projetos de desenvolvimento de software }(2 ; 2 ; \\
0,4 \%)\end{array}$ & $\{$ CAT23\} Garantia da Qualidade (GQA) $(2,7 \%)$ \\
\hline $\begin{array}{l}\{\text { RI120\} A organização pode não realizar as } \\
\text { revisões nos marcos do projeto de } \\
\text { desenvolvimento de software }(1 ; 1 ; 0,2 \%)\end{array}$ & $\begin{array}{l}\text { \{RI051\} Grupo de Garantia da Qualidade exerce suas } \\
\text { atividades inadequadamente }(8 ; 8 ; 1,5 \%)\end{array}$ \\
\hline $\begin{array}{l}\{\mathrm{RI} 122\} \mathrm{O} \text { processo definido ter muitos } \\
\text { artefatos }(2 ; 2 ; 0,4 \%)\end{array}$ & $\begin{array}{l}\text { \{RI131\} A organização pode definir a monitoração } \\
\text { dos processos a partir de medidas pouco } \\
\text { significativas }(3 ; 3 ; 0,6 \%)\end{array}$ \\
\hline $\begin{array}{l}\{\text { RI128\} A organização pode não compreender } \\
\text { o que deve ser monitorado }(1 ; 1 ; 0,2 \%)\end{array}$ & $\begin{array}{l}\text { \{RI132\} Dificuldades na identificação, registro e } \\
\text { comunicação dos problemas e não conformidades }(3 ; \\
3 ; 0,6 \%)\end{array}$ \\
\hline \{CAT20\} Gerência de Requisitos $(2,1 \%)$ & \{CAT24\} Medição (MED) $(4,6 \%)$ \\
\hline $\begin{array}{l}\{\text { RI004\} Falta de ferramenta para apoiar a } \\
\text { rastreabilidade de requisitos }(1 ; 1 ; 0,2 \%)\end{array}$ & $\begin{array}{l}\{\mathrm{RI} 062\} \text { A coleta de medidas pode ser realizada de } \\
\text { forma inadequada }(8 ; 8 ; 1,5 \%)\end{array}$ \\
\hline $\begin{array}{l}\{\text { RI014\} Falta de conhecimento do processo de } \\
\text { gerência de requisitos }(1 ; 1 ; 0,2 \%)\end{array}$ & $\begin{array}{l}\{\text { RI063 }\} \text { Não conseguir estabilizar a medição da } \\
\text { produtividade }(1 ; 1 ; 0,2 \%)\end{array}$ \\
\hline $\begin{array}{l}\{\mathrm{RI} 122\} \mathrm{O} \text { processo definido ter muitos } \\
\text { artefatos }(2 ; 2 ; 0,4 \%)\end{array}$ & $\begin{array}{l}\text { RI064\} Os objetivos da medição podem não ser } \\
\text { estabelecidos a partir dos objetivos organizacionais } \\
(2 ; 3 ; 0,6 \%)\end{array}$ \\
\hline $\begin{array}{l}\{\text { RI123\} As mudanças nos requisitos podem } \\
\text { não ser refletidas em todos os produtos }(6 ; 7 ; \\
1,3 \%)\end{array}$ & $\begin{array}{l}\{\text { RI065 }\} \text { Dificuldades na criação do repositório } \\
\text { organizacional de medições }(1 ; 1 ; 0,2 \%)\end{array}$ \\
\hline $\begin{array}{c}\text { \{CAT21\} Gerência de Configuração (GCO) } \\
(\mathbf{1 , 0 \% )}\end{array}$ & $\begin{array}{l}\text { \{RI066\} As medidas definidas no plano de medição } \\
\text { não trazem nenhum benefício para organização }(3 ; 3 ; \\
0,6 \%)\end{array}$ \\
\hline $\begin{array}{l}\{\text { RI013\} Execução errada de atividades de } \\
\text { gerência de configuração }(4 ; 4 ; 0,8 \%)\end{array}$ & $\begin{array}{l}\{\text { RI067 }\} \text { Não adequação de bases de medidas à } \\
\text { aplicação das técnicas estatísticas }(1 ; 1 ; 0,2 \%)\end{array}$ \\
\hline $\begin{array}{l}\{\mathrm{RI} 124\} \text { Consultoria pode assumir as atividades } \\
\text { da empresa referente à iniciativa de MPS }(1 ; 1 ; \\
0,2 \%)\end{array}$ & $\begin{array}{l}\{\text { RI068 }\} \text { Falta de ferramentas para apoiar o processo } \\
\text { de medição para alta maturidade }(2 ; 2 ; 0,4 \%)\end{array}$ \\
\hline & $\begin{array}{l}\{\text { RI069\} Dificuldades na definição do modelo de } \\
\text { desempenho }(4 ; 4 ; 0,8 \%)\end{array}$ \\
\hline & $\begin{array}{l}\{\text { RI070 }\} \text { Dificuldades na definição do plano de } \\
\text { medição }(1 ; 1 ; 0,2 \%)\end{array}$ \\
\hline
\end{tabular}

Na categoria " $\{$ CAT19\} Gerência de Projetos (GPR)" o risco " $\{$ RI015 $\}$ Falta de conhecimento de práticas de gerência de projetos" foi o mais mencionado com 5 citações. Como consequências desse risco identificaram-se inércia, desânimo e um sentimento de incapacidade pela equipe. Uma ação identificada para tratar esse risco foi a realização de um evento dentro da organização para lembrar a definição da equipe na iniciativa de MPS e ressaltar o apoio e interesse da alta administração.

A categoria " $\{\mathrm{CAT} 20\}$ Gerência de Requisitos (GRE)" apresenta riscos referentes à falta de conhecimento no processo, a quantidade de artefatos gerados e, 
principalmente, o " $\{$ RI123\} As mudanças nos requisitos podem não ser refletidas em todos os produtos" representa a dificuldade em garantir que a rastreabilidade dos requisitos seja de fato institucionalizada. Para tratar esse risco, o responsável pela iniciativa de MPS deve acompanhar formalmente a iniciativa de MPS e, caso seja necessário, deve propor a realização de um novo treinamento reforçando os pontos em que ocorreram o maior número de falhas de execução.

O risco " $\{$ RI013 $\}$ Execução errada de atividades de gerência de configuração" possui a maior frequência da categoria " $\{$ CAT21\} Gerência de Configuração (GCO)". As principais causas identificadas para ocorrência desse risco foram "falta de entendimento dos conceitos"; "mudanças constantes na equipe" e "falta de apoio da consultoria". As principais ações identificadas foram "incentivar o estudo de todo o plano de gerência de configuração" e "capacitar a equipe para cada atividade do plano".

Na categoria " $\{$ CAT22 $\}$ Gerência de Portfólio de Projetos (GPP)" apenas dois riscos foram encontrados. Para o risco mais citado " $\{\mathrm{RI} 125\}$ Um único colaborador pode ser responsável pela análise técnica dos projetos", a causa identificada foi o fato de o colaborador responsável pela perspectiva técnica não ter conhecimento técnico de todos os projetos. A ação de tratamento identificada foi alternar os responsáveis pela perspectiva técnica de acordo com o conhecimento que cada colaborador possui.

Na categoria " $\{$ CAT23\} Garantia da Qualidade (GQA)" o risco mais citado é o " $\{$ RI051\} Grupo de Garantia da Qualidade exerce suas atividades inadequadamente" com 9 citações. Uma ação de tratamento identificada para esse risco é a necessidade de realizar um novo treinamento reforçando os pontos em que ocorrem o maior números de falhas de execução. Duas causas foram identificadas como geradoras desse risco "a garantia da qualidade é realizada por um consultor externo" e "gerentes de projetos, visando aprimorar produtos, fazem alterações no formato dos artefatos e simplificações no processo sem comunicar o Grupo de Garantia da Qualidade".

A categoria " $\{\mathrm{CAT} 24\}$ Medição (MED)" obteve o maior número de citações entre os processos do nível G e F. O risco " $\{$ RI062 $\}$ A coleta de medidas pode ser realizada de forma inadequada" obteve 8 citações e possui 8 eventos associados. Uma consequência identificada é que informações relevantes não são coletadas. Uma ação de tratamento identificada propõe que os líderes do projeto de desenvolvimento devam participar de forma intensiva da medição, auxiliando principalmente na contextualização dos dados e na monitoração dos dados de medição coletados pelos membros da equipe.

A Figura 4 indica que através dos eventos associados ao risco " $\{\mathrm{RI} 062\}$ " é possível um entendimento mais contextualizado. Esse foi o motivo dos códigos de primeiro nível estarem escritos de uma forma mais abrangente. Assim, um profissional que utilize o estudo poderá compreender o risco de uma maneira mais genérica através das categorias e de um forma mais detalhada através dos eventos, causas e consequências. 


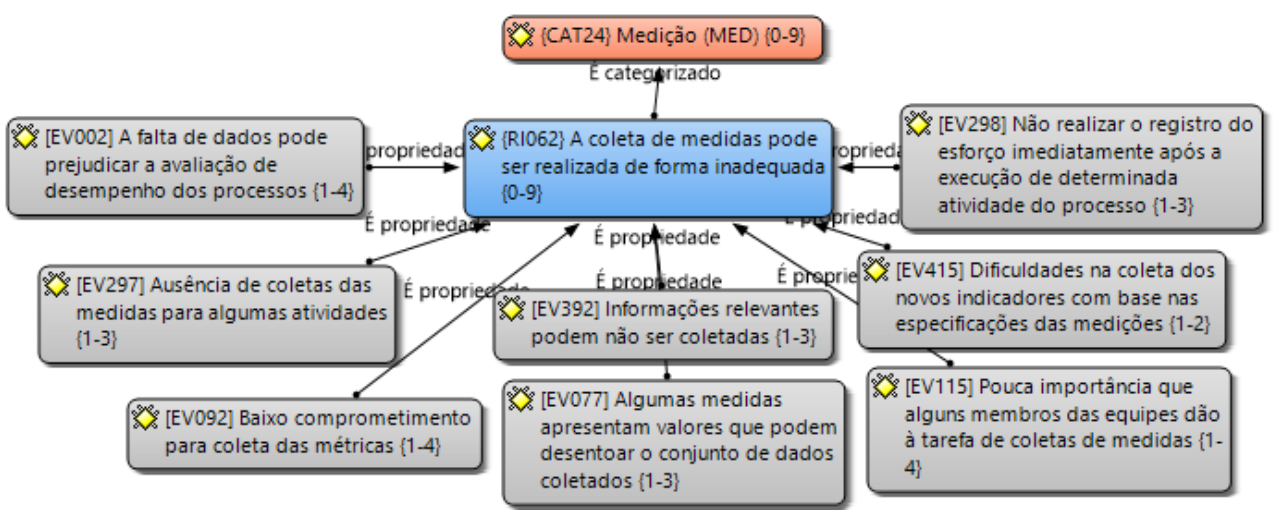

Figura 4. Eventos Associados ao Risco $\{$ RI062\}

\section{Comparação com Riscos Apresentados em Trabalhos Relacionados}

Niazi (2012) analisou publicações internacionais e realizou um survey com profissionais australianos. Dezoito riscos foram identificados e analisados em termos de ocorrência, sendo eles: Mudança na mentalidade dos gerentes e da equipe técnica; Inércia; Equipe inexperiente / Falta de conhecimento; Falta de consciência; Falta de comunicação; Falta da definição de uma metodologia para implantação da iniciativa de MPS; Falta de gerenciamento de projetos; Falta de recursos; Falta de patrocínio; Falta de apoio; Falta de ferramentas; Falta de treinamento; Experiências negativas ou ruins; Políticas organizacionais; Excesso de documentação / Procedimentos Formais; A MPS atrapalha o trabalho; Rotatividade de pessoal e Pressão de tempo.

Todos os riscos identificados por Niazi (2012) também foram identificados neste estudo, entretanto, não é possível realizar comparações na quantidade de citações, pois os trabalhos utilizaram amostras e métodos de contagem diferentes. Alguns riscos encontrados neste estudo não foram identificados em Niazi (2012). Por exemplo, as categorias de riscos referentes ao planejamento (categorias de " $\{$ CAT12 $\}$ " a " $\{$ CAT1 1$\} ")$ da iniciativa e da avaliação (categorias " $\{$ CAT33\}" e "\{CAT34\}"), assim como os específicos por processo (categorias de "\{CAT19\}" a "\{CAT32\}") e do modelo de negócios do MPS.BR de implantação cooperada (categoria " $\{$ CAT14\}").

Não é possível realizar uma comparação com o estudo de Mendes et al. (2007), citado na revisão da literatura, devido ao fato de o artigo em questão ter entrado no escopo do mapeamento sistemático.

\section{Conclusões e Trabalhos Futuros}

Este trabalho apresentou o resultado parcial de uma pesquisa qualitativa com o objetivo de identificar riscos que podem afetar iniciativas de melhoria de processos de software. Um mapeamento sistemático foi conduzido a partir da análise dos artigos do WAMPS e SBQS. No total, 87 artigos fizeram parte do escopo. Após aplicação de procedimentos de codificação, foram identificados 1152 códigos: 398 eventos, 159 causas, 173 consequências, 302 ações de tratamento e 120 efeitos. Os eventos foram agrupados em riscos e os riscos foram agrupados em categorias de risco. Ao final dessa codificação, foram identificados 134 riscos em 34 categorias. A principal contribuição deste trabalho foi a apresentação e análise de uma lista parcial de riscos que podem afetar a definição e a implantação de processos do nível G e F do MR-MPS-SW. Devido à restrição de 
espaço não foi possível apresentar todos os eventos, causas, consequências e ações de tratamento identificados, apenas os riscos mais críticos, isto é, os que apresentam o maior número de citações foram analisados através de suas causas, consequências e algumas ações de tratamento.

Embora apenas as categorias de definição, implantação e de processos específicos tenham sido apresentadas neste artigo, as demais categorias identificadas não devem ser ignoradas. Destaca-se ainda, que as porcentagens indicadas neste estudo não representam juízo de valor ou o impacto na iniciativa de MPS. Cada iniciativa, possui restrições e contextos diferentes. Entretanto, as categorias e riscos identificados podem servir como uma lista inicial para customização de um plano de riscos para uma iniciativa de MPS. Estudos qualitativos permitem diferentes interpretações dos pesquisadores (Corbin e Strauss, 2008). Portanto, este estudo realizou as seguintes ações para tratar as limitações dos resultados (Recker, 2013): (i) o estudo foi auditado por outros pesquisadores e poucas divergências foram encontradas (confiabilidade e confirmabilidade); (ii) foi realizada uma triangulação dos resultados com Niazi (2012) (credibilidade); (iii) a codificação através de uma descrição detalhada dos contextos dos riscos por meio dos eventos, causas e consequências permitem que os resultados possam ser usados em outros contextos (validade externa). Entretanto, destaca-se que essa pesquisa não tem intenção de gerar uma teoria substantiva ou estabelecer hipóteses.

A próxima investigação deste trabalho será a realização de estudos de caso para avaliar se os elementos identificados (categoria de risco, risco, causa, consequência, ação de tratamento e efeito) são adequadas como entrada para um processo de identificação de riscos de uma iniciativa de melhoria de processos de software.

\section{Agradecimentos}

Os autores agradecem à CAPES e à FAPERJ (projeto E-26/110.438/2014) pelo auxílio financeiro. Também agradecemos a Bianca Trinkenreich, Diego Cruz, Raphael Khoury, Patrícia Lima e a Profa. Tayana Conte por suas valiosas contribuições.

\section{Referências}

Bandeira-de-Melo, R. e Cunha, C. J. C. (2010) "Grounded Theory". In: Godoi, C. K. Bandeira-de-Melo, R., Silva, A. B. (Org.) "Pesquisa qualitativa em Estudos Organizacionais: Paradigmas, Estratégias e Métodos”, $2^{\circ}$ edição, São Paulo, Saraiva.

Birk, A. and Pfahl, D. (2002) "A Systems Perspective on Software Process Improvement". Em: Product Focused Software Process Improvement, 2002, pp 418., v. 2559.

CMMI Product Team (2010) CMMI ${ }^{\circledR}$ for Development (CMMI-DEV), V1.3, Software Engineering Institute. Disponível em: http://www.sei.cmu.edu/. Último acesso em: 01/04/2015.

Corbin, J. and Strauss, A. (2008) "Basics of Qualitative Research: Techniques and Procedures for Developing Grounded Theory". 3rd. London, SAGE Publications.

Dyba, T. (2002) "Enabling Software Process Improvement: An Investigation of the Importance of Organizational Issues.” Empirical Software Engineering 7 (4): 387 90.

Falbo, R. A. (2010). Uma Ontologia de Riscos de Software IX Simpósio Brasileiro de Qualidade de Software (SBQS2010), Belém, Para, pp. 151-156 
ISO (2009) 31000, Risk Management-Principles and Guidelines. Geneva: International Standards Organization.

Iversen, J., Mathiassen, L., Axel, N. (2004) "Managing Risk in SPI: AN Action Research Approach.” MIS Quarterly: Manag. Inform. Systems 28 (3): 395-434.

Kitchenham, B. e Charters, S. (2007) "Guidelines for performing Systematic Literature Reviews in Software Engineering", Technical Report EBSE 2007-001, Keele University and Durham University Joint Report.

Mendes F., Almeida J., Arruda E. (2011) "Experiência de Implantação de Melhoria de Processos de Software em um Laboratório de Pesquisa". VII Workshop Anual do MPS, Campinas, SP, 2011, pp 114-123.

Mendes, F. F., Oliveira, J. L., Fernandes, P. G., et al. (2007) "Análise de Riscos na Implantação de MPS”. ProQualiti - Qualidade na Produção de Software Vol.3, Num.3, Novembro, 2007, pp 25-32.

Niazi, M. (2012) "An Exploratory Study of Software Process Improvement Implementation Risks". Journal of Software Evolution and Process 24 (8): 877-94.

Niazi, M., Wilson, D., Zowghi, D. (2006) "Critical success factors for SPI implementation: an empirical study", Software Process: Improvement and Practice, v. 11, n. 2 (Mar), pp. 193-211.

O'Connor, R. and Coleman, G. (2007) "An investigation of barriers to the adoption of software process best practice models". 18th Australasian Conference on Information Systems, Toowoomba, Australia, pp. 780-789.

Paulk, M., Goldenson, D., White, D. (2000) "The 1999 Survey of High Maturity Organizations", Soft. Eng. Institute, Carnegie Mellon University, Pittsburgh, PA.

PMI - Project Management Institute. (2013) "Um Guia do Conjunto de Conhecimentos em Gerenciamento de Projetos: Guia PMBOK”. Pensilvânia: PMI, Quinta edição.

Rainer, A. and Hall, T. (2003) "A quantitative and qualitative analysis of factors affecting software processes". Journal of Systems and Software, v. 66, n. 1, pp. 7-21.

Recker, J. (2012) "Scientific Research in Information Systems: A Beginner's Guide", Queensland, Australia, Springer.

Rocha, R., Montoni, M., Santos, G., et al. (2005) "Dificuldades e Fatores de Sucesso na Implementação de Processos de Software Utilizando o MR-MPS e o CMMI". I Encontro de Implementadores do MPS.BR, Brasília, Brasil.

Rodrigues, J. e Kirner, T. (2010). "Benefícios, Fatores de Sucesso e Dificuldades da Implantação do Modelo MPS.BR", IX Simpósio Brasileiro de Qualidade de Software (SBQS2010), Belém, Para, pp. 41-56.

Santos, G. (2011) "Influência e Impacto do Programa MPS.BR na Pesquisa Relacionada à Qualidade de Software no Brasil", X Simpósio Brasileiro de Qualidade Software (SBQS 2011), Curitiba, Paraná, pp. 73-87.

SOFTEX (2012) "MPS.BR - Guia Geral MPS de Software". Disponível em http://www.softex.br/mpsbr/guias/. Acesso em 01/04/2015.

SOFTEX (2015) "Avaliações MPS-SW (Software) Publicadas (prazo de validade: 3 anos)". Disponível em http://www.softex.br/mpsbr/avaliacoes/mps-sw/. Acesso em $01 / 04 / 2015$. 